\title{
Euler and Laplace integral representations of GKZ hypergeometric functions I
}

\author{
By Saiei-Jaeyeong MatsubarA-HeO \\ Graduate School of Science, Kobe University, 1-1 Rokkodai, Nada-ku, Kobe 657-8501, Japan \\ (Communicated by Masaki Kashiwara, M.J.A., Oct. 12, 2020)
}

\begin{abstract}
We introduce an interpolation between Euler integral and Laplace integral: Euler-Laplace integral. We claim that, when parameters $\delta$ of the integrand are non-resonant, the $\mathcal{D}$-module corresponding to Euler-Laplace integral is naturally isomorphic to GKZ hypergeometric system $M_{A}(\delta)$ where $A$ is a generalization of Cayley configuration. As a topological counterpart of this isomorphism, we establish an isomorphism between certain rapid decay homology group and holomorphic solutions of $M_{A}(\delta)$.
\end{abstract}

Key words: GKZ hypergeometric systems; integral representations; twisted Gauß-Manin connections.

1. Introduction. GKZ hypergeometric system $M_{A}(\delta)$ is a system of linear partial differential equations introduced by I. M. Gel'fand, M. I. Graev, M. M. Kapranov, and A. V. Zelevinskiu in [GGZ87] and [GZK89]. This system is determined by two inputs: a $d \times n(d<n)$ integer matrix $A=(\mathbf{a}(1)|\cdots| \mathbf{a}(n))=\left(a_{i j}\right)$ and a parameter vector $\delta \in \mathbf{C}^{d \times 1}$. GKZ system $M_{A}(\delta)$ is defined by

$$
\begin{cases}E_{i} \cdot f(z)=0 & (i=1, \ldots, n) \\ \square_{u} \cdot f(z)=0 & \left(u={ }^{t}\left(u_{1}, \ldots, u_{n}\right) \in L_{A}\right),\end{cases}
$$

where $L_{A}=\operatorname{Ker}\left(A \times: \mathbf{Z}^{n \times 1} \rightarrow \mathbf{Z}^{d \times 1}\right)$ and $E_{i}$ and $\square_{u}$ are differential operators defined by $E_{i}=$ $\sum_{j=1}^{n} a_{i j} z_{j} \frac{\partial}{\partial z_{j}}+\delta_{i} \quad$ and $\quad \square_{u}=\prod_{u_{j}>0}\left(\frac{\partial}{\partial z_{j}}\right)^{u_{j}}-$ $\prod_{u_{j}<0}\left(\frac{\partial}{\partial z_{j}}\right)^{-u_{j}}$. Throughout this paper, we assume an additional condition $\mathbf{Z} A \stackrel{\text { def }}{=} \mathbf{Z} \mathbf{a}(1)+\cdots+\mathbf{Z} \mathbf{a}(n)=$ $\mathbf{Z}^{d \times 1}$. Writing $\mathcal{D}_{\mathbf{A}^{n}}$ for the Weyl algebra on $\mathbf{A}^{n}$ and $H_{A}(\delta)$ for the left ideal of $\mathcal{D}_{\mathbf{A}^{n}}$ generated by the differential operators (1), we also call the left

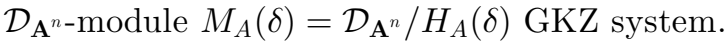

There have been discussed Euler and Laplace integral representations of GKZ systems by several authors ([GKZ90], [ET15]). In this paper, we introduce an integral representation which generalizes both Euler and Laplace integral representations: Euler-Laplace integral representation. In the language of $\mathcal{D}$-modules, integral representations can

2010 Mathematics Subject Classification. Primary 33D70; Secondary 32C 38 . be defined as direct images of integrable connections commonly referred to as Gauß-Manin connections. Therefore, we need to establish an isomorphism between GKZ system and a certain Gauß-Manin connection. This is formulated in $\S 2$. In $\S 3$, we describe the space of rapid decay cycles generalizing the argument of [ET15]. The detailed argument is quite technical and we only outline the construction of the toric compactification. The readers can find proofs in $[\mathrm{MHa}, \S 2, \S 3]$.

2. Gauß-Manin connections and GKZ $\mathcal{D}$-modules. We follow the notation of [HTT08]. Let $\quad h_{l, z^{(l)}}(x)=\sum_{j=1}^{N_{l}} z_{j}^{(l)} x^{\mathbf{a}^{(l)}(j)} \quad(l=0,1, \ldots, k) \quad$ be Laurent polynomials on the algebraic torus $\left(\mathbf{G}_{m}\right)_{x}^{n}$. Here, the subindex $x$ means that we use the symbol $x$ as a coordinate of the torus. The coefficients $z=$ $\left(z_{j}^{(l)}\right)_{j, l}$ are regarded as independent variables of the affine space $\mathbf{A}_{z}^{N}$ with $N=N_{0}+\cdots+N_{k}$. We put $X_{0}=\mathbf{A}_{z}^{N} \times\left(\mathbf{G}_{m}\right)_{x}^{n} \backslash\left\{(z, x) \in \mathbf{A}^{N} \times\left(\mathbf{G}_{m}\right)^{n} \mid\right.$ $\left.h_{1, z^{(1)}}(x) \cdots h_{k, z^{(k)}}(x)=0\right\}$ and write $\pi: X_{0} \rightarrow \mathbf{A}_{z}^{N}$ for the natural projection. The Euler-Laplace integral representation is defined as a complex of $\mathcal{D}$-modules $\int_{\pi} \mathcal{O}_{X_{0}} e^{h_{0, z^{(0)}}(x)} h_{1, z^{(1)}}(x)^{-\gamma_{1}} \cdots h_{k, z^{(k)}}(x)^{-\gamma_{k}} x^{c}$ where $\gamma_{l} \in$ $\mathbf{C}$ and $c \in \mathbf{C}^{n \times 1}$ are parameters.

The following theorem proves the equivalence of Laplace integral representation and EulerLaplace integral representation.

Theorem 2.1 (Cayley trick for Euler-Laplace integrals). We put $X_{k}=\mathbf{A}_{z}^{N} \times\left(\mathbf{G}_{m}\right)_{y}^{k} \times\left(\mathbf{G}_{m}\right)_{x}^{n}$. Let $\varpi: X_{k} \rightarrow \mathbf{A}_{z}^{N}$ be the natural projection and 
$\gamma_{l} \in \mathbf{C} \backslash \mathbf{Z}, \delta \in \mathbf{C}^{n \times 1}$ be parameters. Then, one has a natural isomorphism

$$
\begin{aligned}
& \int_{\pi} \mathcal{O}_{X_{0}} e^{h_{0, z^{(0)}}(x)} h_{1, z^{(1)}}(x)^{-\gamma_{1}} \cdots h_{k, z^{(k)}}(x)^{-\gamma_{k}} x^{c} \\
& \simeq \int_{\varpi} \mathcal{O}_{X_{k}} y^{\gamma} x^{c} e^{h_{z}(y, x)},
\end{aligned}
$$

where $h_{z}(y, x)=h_{0, z^{(0)}}(x)+\sum_{l=1}^{k} y_{l} h_{l, z^{(l)}}(x)$.

Corollary 2.2. Under the assumption of Theorem 2.1, one has a natural isomorphism

$$
\begin{aligned}
& \int_{\pi !} \mathcal{O}_{X_{0}} e^{h_{0, z^{(0)}}(x)} h_{1, z^{(1)}}(x)^{-\gamma_{1}} \cdots h_{k, z^{(k)}}(x)^{-\gamma_{k}} x^{c} \\
& \simeq \int_{\varpi !} \mathcal{O}_{X_{k}} y^{\gamma} x^{c} e^{h_{z}(y, x)} .
\end{aligned}
$$

We put $\Phi=\Phi(z, x)=e^{h_{0, z^{(0)}}(x)} h_{1, z^{(1)}}(x)^{-\gamma_{1}} \ldots$ $h_{k, z^{(k)}}(x)^{-\gamma_{k}} x^{c}, \Phi_{k}=y^{\gamma} x^{c} e^{h_{z}(y, x)}$ to simplify the notation. Let us formulate the main theorem of this section. We define an $n \times N_{l}$ matrix $A_{l}$ by $A_{l}=$ $\left(\mathbf{a}^{(l)}(1)|\cdots| \mathbf{a}^{(l)}\left(N_{l}\right)\right)$. Then, we define the Cayley configuration $A$ as an $(n+k) \times N$ matrix by

$$
A=\left(\begin{array}{c|c|c|c|c}
0 \cdots 0 & 1 \cdots 1 & 0 \cdots 0 & \cdots & 0 \cdots 0 \\
\hline 0 \cdots 0 & 0 \cdots 0 & 1 \cdots 1 & \cdots & 0 \cdots 0 \\
\hline \vdots & \vdots & \vdots & \ddots & \vdots \\
\hline 0 \cdots 0 & 0 \cdots 0 & 0 \cdots 0 & \cdots & 1 \cdots 1 \\
\hline A_{0} & A_{1} & A_{2} & \cdots & A_{k}
\end{array}\right) .
$$

Combining Theorem 2.1 with the result of [SW09], we can obtain the following main result of this section.

Theorem 2.3. Suppose that the parameter $\delta={ }^{t}\left(\gamma_{1}, \ldots, \gamma_{k}, c\right)$ is non-resonant and $\gamma_{l} \notin \mathbf{Z}$ for $l=1, \ldots, k$. Then, one has a sequence of canonical isomorphisms of $\mathcal{D}_{\mathbf{A}_{z}^{N}-\text { modules }}$

$$
M_{A}(\delta) \simeq \int_{\varpi} \mathcal{O}_{X_{k}} \Phi_{k} \simeq \int_{\pi} \mathcal{O}_{X_{0}} \Phi
$$

Moreover, the regularization conditions

$$
\int_{\varpi} \mathcal{O}_{X_{k}} \Phi_{k} \simeq \int_{\varpi !} \mathcal{O}_{X_{k}} \Phi_{k}
$$

and

$$
\int_{\pi} \mathcal{O}_{X_{0}} \Phi \simeq \int_{\pi !} \mathcal{O}_{X_{0}} \Phi
$$

hold.

In the proof of the last two isomorphisms of Theorem 2.3, we use the proper direct image description of Fourier transform of $\mathcal{D}$-modules
([Dai00, Proposition 2.2.3.2]).

3. Description of the rapid decay homology groups of Euler-Laplace integrals. We formulate the isomorphism between the space of holomorphic solutions of GKZ system and a certain rapid decay homology group. To begin with, let us remark that there is a concrete version of the isomorphism (5).

Theorem 3.1. There is an isomorphism

$$
\int_{\pi}^{0} \mathcal{O}_{X_{0}} \Phi \rightarrow \int_{\varpi}^{0} \mathcal{O}_{X_{k}} \Phi_{k},
$$

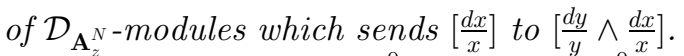

Here, we realize $\int_{\pi}^{0} \mathcal{O}_{X_{0}} \Phi$ and $\int_{\varpi}^{0} \mathcal{O}_{X_{k}} \Phi_{k}$ in terms of relative de Rham cohomology groups and we set $\frac{d x}{x}=\frac{d x_{1}}{x_{1}} \wedge \cdots \wedge \frac{d x_{n}}{x_{n}}$ and $\frac{d y}{y}=\frac{d y_{1}}{y_{1}} \wedge \cdots \wedge \frac{d y_{k}}{y_{k}}$.

Corollary 3.2. If the parameter $\delta$ is nonresonant and $\gamma_{l} \notin \mathbf{Z}$ for any $l=1, \ldots, k, M_{A}(\delta) \ni$ $[1] \mapsto\left[\frac{d x}{x}\right] \in \int_{\pi}^{0} \mathcal{O}_{X_{0}} \Phi$ defines an isomorphism of $\mathcal{D}_{\mathbf{A}^{N} \text {-modules. }}$

The proof of Theorem 3.1 is based on tedious calculations of relative de Rham complexes.

Now we discuss the solutions of Laplace-GaussManin connection $\int_{\pi} \mathcal{O}_{X_{0}} \Phi$. We repeat the relevant material from [ET15] and [Hie09] without proofs. Let $U$ be a smooth complex affine variety, let $f$ : $U \rightarrow \mathbf{A}^{1}$ be a non-constant morphism, and let $M=$ $(E, \nabla)$ be a regular integrable connection on $U$. We consider an embedding of $U$ into a smooth projective variety $X$ with a meromorphic prolongation $\bar{f}: X \rightarrow \mathbf{P}^{1}$ of $f$. We assume that $D=X \backslash U$ is a normal crossing divisor. Any projective variety $X$ with these conditions is called a good compactification. We decompose $D$ as $D=f^{-1}(\infty) \cup D_{i r r}$. Then, we write $\widehat{X^{D}}=\widetilde{X}$ for the real oriented blow-up of $X$ along $D$ and write $\pi_{X}: \widetilde{X} \rightarrow X$ for the associated morphism ([Sab13, §8.2]). Let $\widetilde{\mathbf{P}^{1}}$ denote the real oriented blow-up of $\mathbf{P}^{1}$ at infinity and $\pi_{\infty}: \widetilde{\mathbf{P}^{1}} \rightarrow \mathbf{P}^{1}$ denote the associated morphism. Note that the closure of the ray $[0, \infty) e^{\sqrt{-1 \theta}}$ in $\widetilde{\mathbf{P}^{1}}$ and $\widetilde{\mathbf{P}^{1}} \backslash \mathbf{C}$ has a unique intersection point which we will denote by $e^{\sqrt{-1} \theta} \infty$. Now, a morphism $\tilde{f}: \widetilde{X} \rightarrow X$ is naturally induced so that it fits into a commutative diagram

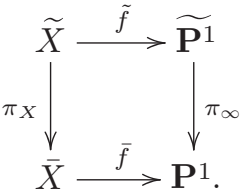

We set $\quad \widetilde{D^{r . d}}=\tilde{f}^{-1}\left(\left\{e^{\sqrt{-1} \theta} \infty \mid \theta \in\left(\frac{\pi}{2}, \frac{3 \pi}{2}\right)\right\}\right) \backslash$ $\pi_{X}^{-1}\left(D_{i r r}\right) \subset \widetilde{X} . \quad$ We $\quad$ put $\quad \mathcal{L}=\left(\operatorname{Ker}\left(\nabla^{a n}\right.\right.$ : 
$\left.\left.\mathcal{O}_{X^{a n}}\left(E^{a n}\right) \rightarrow \Omega_{X^{a n}}^{1}\left(E^{a n}\right)\right)\right)^{\vee}$, where ${ }^{\vee}$ stands for the dual local system. We consider the natural inclusion $U^{a n} \stackrel{j}{\hookrightarrow} U^{a n} \cup \widetilde{D^{r . d}}$. Then, the rapid decay homology group of M. Hien $\mathrm{H}_{*}^{r . d} \cdot\left(U^{a n},\left(M e^{f}\right)^{\vee}\right)$ is defined in this setting by

$$
\mathrm{H}_{*}^{r . d .}\left(U^{a n},\left(M e^{f}\right)^{\vee}\right)=\mathrm{H}_{*}\left(U^{a n} \cup \widetilde{D^{r . d} .}, \widetilde{D^{r . d} .} ; j_{*} \mathcal{L}\right)
$$

([Hie09], see also [ET15] and [MHb]). Note that $U^{a n} \cup \widetilde{D^{r . d}}$ is a topological manifold with boundary and that $j_{*} \mathcal{L}$ is a local system on $U^{a n} \cup \widetilde{D^{r . d}}$. We set $\mathrm{H}_{d R}^{*+\operatorname{dim} U}\left(U, M e^{f}\right)=\mathrm{H}^{*}\left(\operatorname{DR}_{U / \mathrm{pt}}\left(M e^{f}\right)\right)$. The main result of [Hie09] states that the period pairing $\mathrm{H}_{*}^{r . d .}\left(U^{a n},\left(M e^{f}\right)^{\vee}\right) \times \mathrm{H}_{d R}^{*}\left(U, M e^{f}\right) \rightarrow \mathbf{C}$ is perfect.

Remark 3.3. We put $\widetilde{D_{0}^{r . d} .}=$ $\tilde{f}^{-1}\left(\left\{e^{\sqrt{-1} \theta} \infty \mid \theta \in\left(\frac{\pi}{2}, \frac{3 \pi}{2}\right)\right\}\right)$ and write $\bar{j}$ for the nat-

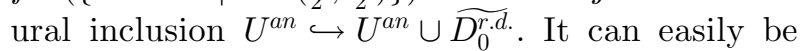
seen that the inclusion $\left(U^{a n} \cup \widetilde{D^{r . d} .}, \widetilde{D^{r . d} .}\right) \hookrightarrow\left(U^{a n} \cup\right.$ $\widetilde{D_{0}^{r . d}}, \widetilde{D_{0}^{r . d} .}$ ) is a homotopy equivalence ([MHb, Lemma 2.3]). Therefore, the rapid decay homology group can be computed by the formula $\mathrm{H}_{*}^{r . d .}\left(U^{a n},\left(M e^{f}\right)^{\vee}\right)=\mathrm{H}_{*}\left(U^{a n} \cup \widetilde{D_{0}^{r . d}}, \widetilde{D_{0}^{r . d} .} ; \bar{j}_{*} \mathcal{L}\right)$. Note that this realization is compatible with the period pairing.

We construct a family of good compactifications $X$ associated to the Laplace-Gauss-Manin connection $\int_{\pi}^{0} \mathcal{O}_{X_{0}} \Phi$. First, we write $\Delta_{0}$ for the convex hull of the set $\left\{\mathbf{a}^{(0)}(1), \ldots, \mathbf{a}^{(0)}\left(N_{0}\right)\right\}$ and the origin and write $\Delta_{l}$ for the convex hull of the set $\left\{\mathbf{a}^{(l)}(1), \ldots, \mathbf{a}^{(l)}\left(N_{l}\right)\right\} \quad(l=1, \ldots, k)$. For any covector $\xi \in\left(\mathbf{R}^{n}\right)^{*}$, we set $\Delta_{l}^{\xi}=\left\{v \in \Delta_{l} \mid\langle\xi, v\rangle=\right.$ $\left.\min _{w \in \Delta_{l}}\langle\xi, w\rangle\right\}$ and $h_{l, z^{(l)}}^{\xi}(x)=\sum_{\mathbf{a}^{(l)}(j) \in \Delta_{l}^{\xi}} z_{j} x^{\mathbf{a}^{(l)}(j)}$. Now, we consider the dual fan $\Sigma$ of the Minkowski sum $\Delta_{0}+\Delta_{1}+\cdots+\Delta_{k}$. By taking a refinement if necessary, we may assume that $\Sigma$ is a smooth fan. Then, the associated toric variety $X=X(\Sigma)$ is sufficiently full for any $\Delta_{l}$ in the sense of [Hov77]. We write $\left\{D_{j}\right\}_{j \in J}$ for the set of torus invariant divisors of $X$.

Definition 3.4. We say that a point $z=$ $\left(z^{(0)}, z^{(1)}, \ldots, z^{(k)}\right) \in \mathbf{C}^{N}$ is nonsingular if the following two conditions are both satisfied:

(a) For any $1 \leq l_{1}<\cdots<l_{s} \leq k$, the Laurent polynomials $h_{l_{1}, z^{\left(l_{1}\right)}}(x), \ldots, h_{l_{s}, z^{\left(l_{s}\right)}}(x)$ are nonsingular in the sense of [Hov77], i.e., for any covector $\xi \in\left(\mathbf{R}^{n}\right)^{*}$, the $s$-form $d_{x} h_{l_{1}, z^{\left(l_{1}\right)}}^{\xi}(x) \wedge \cdots \wedge d_{x} h_{l_{s}, z^{\left(l_{s}\right)}}^{\xi}(x)$ never vanishes on the set $\left\{x \in\left(\mathbf{C}^{\times}\right)^{n} \mid h_{l_{1}, z^{\left(l_{1}\right)}}^{\xi}(x)=\cdots=\right.$ $\left.h_{l_{s}, z^{\left(l_{s}\right)}}^{\xi}(x)=0\right\}$. (b) For any covector $\xi \in\left(\mathbf{R}^{n}\right)^{*}$ such that $0 \notin \Delta_{0}^{\xi}$ and for any $1 \leq l_{1}<\cdots<l_{s} \leq k$ ( $s$ can be 0$)$, the $(s+1)$-form $d h_{0, z^{(0)}}^{\xi}(x) \wedge d h_{l_{1}, z_{1}^{\left(l_{1}\right)}}^{\xi}(x) \wedge \cdots \wedge$ $d h_{l_{s}, z^{\left(l_{s}\right)}}^{\xi}(x)$ never vanishes on the set $\{x \in$ $\left.\left(\mathbf{C}^{\times}\right)^{n} \mid h_{l_{1}, z^{\left(l_{1}\right)}}^{\xi}(x)=\cdots=h_{l_{s}, z^{\left(l_{s}\right)}}^{\xi}(x)=0\right\}$.

Remark 3.5. If $k=0$, the nonsingularity condition is equivalent to the non-degenerate condition of [Ado94, p.274]. In general, nonsingularity condition is stronger than non-degenerate condition. Nevertheless, it is still a Zariski open dense condition. Note that this is proved in the case when $h_{0, z^{(0)}} \equiv 1$ in [Hov77] (see also [Oka97, Chapter V, COROLLARY (3.2.1)]).

In the following, we fix a nonsingular $z$ and a small positive real number $\varepsilon$. Let $\Delta(z ; \varepsilon)$ denote the disk with center at $z$ and with radius $\varepsilon$. By abuse of notation, we write $D_{j}$ for the product $\Delta(z ; \varepsilon) \times D_{j}$. For any $l=0, \ldots, k$, we set $Z_{l}=$ $\overline{\left\{\left(z^{\prime}, x\right) \in \Delta(z ; \varepsilon) \times\left(\mathbf{C}^{\times}\right)_{x}^{n} \mid h_{l, z^{(l)}}(x)=0\right\}} \subset \Delta(z ; \varepsilon) \times$ $X$.

Now we take a small positive real number $\varepsilon$ and consider the canonical projection $p: \Delta(z ; \varepsilon) \times X \rightarrow$ $\Delta(z ; \varepsilon)$. Following [ET15], we consider a sequence of blow-ups along codimension 2 divisors $Z_{0} \cap D_{j}$ for $D_{j}$ contained in the pole divisor of $h_{0, z^{\prime(0)}}(x)$. If the pole order of $h_{0, z^{\prime(0)}}(x)$ along $D_{j}$ is $m_{j} \in \mathbf{Z}_{>0}$, one needs at most $m_{j}$ blow-ups along $Z_{0} \cap D_{j}$. Repeating this process finitely many times, we obtain a non-singular complex manifold $\bar{X}$. We write $\bar{p}$ : $\bar{X} \rightarrow \Delta(z ; \varepsilon)$ for the composition of the natural morphism $\bar{X} \rightarrow \Delta(z ; \varepsilon) \times X$ with the canonical projection $\Delta(z ; \varepsilon) \times X \rightarrow \Delta(z ; \varepsilon)$. We also write $\bar{Z}_{l}$ and $\bar{D}_{j}$ for the proper transforms of $Z_{l}$ and $D_{j}$. We equip $\bar{X}$ with the Whitney stratification coming from the normal crossing divisor $\bar{D}=\left\{\bar{Z}_{l}\right\}_{l=1}^{k} \cup$ $\left\{\bar{D}_{j}\right\}_{j \in J} \cup\{$ exceptional divisors of blow-ups $\}$. We have a diagram $\Delta(z ; \varepsilon) \stackrel{\bar{p}}{\leftarrow} \bar{X} \stackrel{\bar{h}_{0, z^{(0)}}}{\longrightarrow} \mathbf{P}^{1}$. Let us consider a real oriented blow-up $\widetilde{X}=\overline{\bar{X}}^{\bar{D}}$ of $\bar{X}$ along $\bar{D}$. We naturally have the following commutative diagram

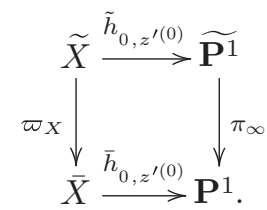

We can show that $\bar{p}^{-1}\left(z^{\prime}\right)$ is a good compactification of $\pi^{-1}\left(z^{\prime}\right)$ for any $z^{\prime} \in \Delta(z ; \varepsilon)$. We define $\widetilde{D^{r . d} .} \subset \widetilde{X}$ by the formula $\widetilde{D^{r . d}}=\tilde{h}_{0, z^{\prime}(0)}^{-1}\left(\left(\frac{\pi}{2}, \frac{3 \pi}{2}\right) \infty\right)$, put $\tilde{p}=$ 
$p \circ \varpi_{X}$ and put $\widetilde{D_{z}^{r . d}}=\widetilde{D^{r . d} .} \cap \tilde{p}^{-1}(z)$. For any $z^{\prime} \in \mathbf{A}^{N}$, let $\Phi_{z^{\prime}}$ denote the multivalued function on $\pi^{-1}\left(z^{\prime}\right)$ defined by $\pi^{-1}\left(z^{\prime}\right) \ni x \mapsto \Phi\left(z^{\prime}, x\right)$. Writ-

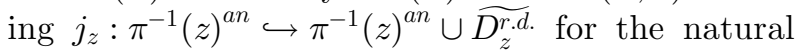
inclusion, we set

$$
\mathrm{H}_{*, z}^{r . d .}=\mathrm{H}_{*}\left(\pi^{-1}(z)^{a n} \cup \widetilde{D_{z}^{r . d}} . \widetilde{D_{z}^{r . d}} ; j_{z *}\left(\underline{\mathbf{C}} \Phi_{z}\right)\right) .
$$

Theorem 3.6. Suppose the parameter vector $\delta$ is non-resonant and $\gamma_{l} \notin \mathbf{Z}$ for any $l=1, \ldots, k$. Suppose that $z \in \mathbf{C}^{N}$ is nonsingular. Then the morphism

$$
\mathrm{H}_{n, z}^{r . d .} \stackrel{\int}{\rightarrow} \operatorname{Hom}_{\mathcal{D}_{\mathbf{C}_{z}^{N}}}\left(M_{A}(\delta), \mathcal{O}_{\mathbf{C}^{N}}\right)_{z}
$$

given by

$$
[\Gamma] \mapsto \int_{\Gamma} \Phi \frac{d x}{x}
$$

is an isomorphism of $\mathbf{C}$-vector spaces.

Remark 3.7. Let $\Omega$ denote the Zariski open dense subset of $\mathbf{A}^{N}$ consisting of nonsingular points. It is straightforward to construct a local system $\mathcal{H}_{n}^{\text {r.d. }}=\bigcup_{z \in \Omega^{a n}} \mathrm{H}_{n, z}^{\text {r.d. }} \rightarrow \Omega^{a n}$ and an isomorphism $\mathcal{H}_{n}^{r . d .} \stackrel{\int}{\rightarrow} \operatorname{Hom}_{\mathcal{D}_{\mathbf{C}_{z}^{N}}}\left(M_{A}(\delta), \mathcal{O}_{\mathbf{C}^{N}}\right)_{\Omega^{a n}}$ whose stalks are identical with (13). See [HR08].

\section{References}

[ Ado94] A. Adolphson, Hypergeometric functions and rings generated by monomials, Duke Math. J. 73 (1994), no. 2, 269-290.

[ Dai00 ] L. Daia, La transformation de Fourier pour les $\mathcal{D}$-modules, Ann. Inst. Fourier (Grenoble) 50 (2000), no. 6, 1891-1944 (2001).

[ ET15 ] A. Esterov and K. Takeuchi, Confluent A-hypergeometric functions and rapid decay homology cycles, Amer. J. Math. 137 (2015), no. 2, 365-409.

[GGZ87] I. M. Gel'fand, M. I. Graev and A. V.
Zelevinskiǔ, Soviet Math. Dokl. 36 (1988), no. 1, 5-10; translated from Dokl. Akad. Nauk SSSR 295 (1987), no. 1, 14-19.

[GKZ90] I. M. Gel'fand, M. M. Kapranov and A. V. Zelevinsky, Generalized Euler integrals and $A$-hypergeometric functions, Adv. Math. 84 (1990), no. 2, 255-271.

[GZK89] I. M. Gel'fand, A. V. Zelevinskiǔ and M. M. Kapranov, Funct. Anal. Appl. 23 (1989), no. 2, 94-106; translated from Funktsional. Anal. i Prilozhen. 23 (1989), no. 2, 12-26.

[ Hie09 ] M. Hien, Periods for flat algebraic connections, Invent. Math. 178 (2009), no. 1, 122 .

[ Hov77 ] A. G. Hovanskiu,, Newton polyhedra, and toroidal varieties, Funkcional. Anal. i Priložen. 11 (1977), no. 4, 56-64, 96.

[ HR08 ] M. Hien and C. Roucairol, Integral representations for solutions of exponential GaussManin systems, Bull. Soc. Math. France 136 (2008), no. 4, 505-532.

[HTT08] R. Hotta, K. Takeuchi and T. Tanisaki, D-modules, perverse sheaves, and representation theory, translated from the 1995 Japanese ed. by K. Takeuchi, Progress in Mathematics, 236, Birkhäuser Boston, Inc., Boston, MA, 2008.

[ MHa ] S.-J. Matsubara-Heo, Euler and laplace integral representations of GKZ hypergeometric functions. arXiv:1904.00565.

[ $\mathrm{MHb}$ ] S.-J. Matsubara-Heo, On rapid decay homology of F.Pham, Kôkyûroku Bessatsu B75 (2019), 1-17.

[ Oka97] M. Oka, Non-degenerate complete intersection singularity, Actualités Mathématiques., Hermann, Paris, 1997.

[ Sab13 ] C. Sabbah, Introduction to Stokes structures, Lecture Notes in Mathematics, 2060, Springer, Heidelberg, 2013.

[ SW09 ] M. Schulze and U. Walther, Hypergeometric D-modules and twisted Gauß-Manin systems, J. Algebra 322 (2009), no. 9, 33923409 . 